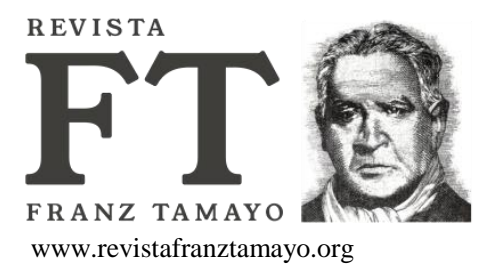

\title{
Violencia: Una perspectiva internacional
}

\author{
Violence: an international perspective
}

Recibido febrero 2021

Arbitrado marzo 2021 Aceptado abril 2021

Publicado mayo 2021

\author{
Antonio Fuguet Smith \\ afsefe@gmail.com \\ Código ORCID: 0000-0002-9722-7695
}

Universidad Pedagógica Experimental Libertador, Caracas, Venezuela

\section{Resumen}

Se estudia la violencia desde una perspectiva social y educacional, especialmente en países de América del Sur, analizados desde las investigaciones internacionales, con enfoque de Educación Comparada. Lo internacional responde a las características de la globalización que permite observar rasgos comunes y peculiaridades. Así, se resalta la manifestación del problema y las preocupaciones, esfuerzos y contribuciones de investigadores a fin de crear estructuras conceptuales propias del problema. Se han tipificado energizantes para una reflexión, concebidos como motores de acción ante el reto que representa la educación hoy. Funcionan como conectores para entender el problema de la violencia y su impacto. La reflexión se realiza desde una búsqueda hermenéutica integrada por tres fuentes: las referidas a los motores, las que abordan el tema de la violencia y la inspiración del autor como sujeto que interactúa con su experiencia y creación con las fuentes, intersubjetivamente. Se organiza este artículo en tres partes: aspectos procedimentales, la derivación de las categorías de análisis y, la instancia de reflexión.

\footnotetext{
Abstract

Violence is studied from a social and educational perspective, especially in South American countries, analyzed from international research, with a comparative education approach. The international perspective responds to the characteristics of globalization that allows us to observe common features and peculiarities. This character highlights the manifestation of the problem and the concerns, efforts and contributions of researchers in order to create conceptual structures specific about the problem. Expressions have been typified as energizing for discussion, conceived as engines of action to face and challenge posed by education today. They function as connectors to understand the problem of violence and its impact. A reflection is carried out from a hermeneutic search composed of three sources: those related to engines, those which address the theme of violence and the inspiration of the author as a subject that interacts with his experience and creation, intersubjectively. In this sense, this article is organized into three parts: procedural aspects, derivation of the categories of analysis and the instance of reflection.
}

\author{
Palabras clave: \\ Violencia; violencia \\ política; violencia \\ académica; \\ violencia \\ ontoepistemometodológica
}

\author{
Keywords: \\ Violence; political \\ violence; academic \\ violence \\ ontoepistemomethodological \\ violence
}




\section{INTRODUCCIÓN}

$\mathrm{E}$ l racional de este artículo es promover oportunidades de reflexión sobre la violencia y sus impactos desde una perspectiva psico-socio-educativa, especialmente en países de América del Sur, analizados desde las perspectivas de investigaciones internacionales, con enfoque de Educación Comparada. Nos ubicamos en una perspectiva internacional dada que las características de globalización o planetización llevan a considerar rasgos comunes sin perder peculiaridades. Este carácter de internacionalidad reviste no sólo la manifestación del problema sino también las preocupaciones de investigadores en el área, sus esfuerzos por estudiar el tema y sus contribuciones a un acervo que permite recrear estructuras conceptuales propias del problema.

Diversos autores han tipificado expresiones que pudieran ser consideradas como energizantes para una discusión sobre la temática. Energizantes en el sentido de lo planteado por Collins (2002) y Fullan (2011) en sus obras, concebidos como motores de acción, especialmente por sus impactos en la tarea de educar. Motores que te hacen, paradójicamente, iluminar y nublar caminos. Desde esta perspectiva se introducen expresiones de Turner (2004), Schoonmaker (2007) y Nucci (2009), sobre el encontrarnos atollados, con una sola talla y pensando en fines que no son suficientes para el retro que representa la educación hoy.

Así que motores y expresiones, como conectores, nos pueden iluminar un poco para poder entender el grave problema social de la violencia y su impacto en entornos y contornos escolares. Por tal motivo, se selecciona un contenido de reflexión, desde una búsqueda hermenéutica integrada por tres fuentes: las referidas a los motores y expresiones, las que abordan el tema de la violencia y la inspiración del autor como sujeto que interactúa con su experiencia y creación con las otras fuentes, en un marco de intersubjetividad. En tal sentido, se organiza, este artículo en tres partes: aspectos procedimentales, recorrido por las fuentes para la derivación de las categorías de análisis y, por último, la instancia de reflexión del autor. 


\section{Aspectos procedimentales}

Fase 1: Selección de conductores, motores y energizantes para facilitar la interpretación

Para Collins (2002), los motores son como un constante flujo de ganancias no exclusivamente material. El ser humano tiene la perspicacia de centrarse con energizantes para el logro de una variedad de aprendizajes. Ellos constituyen motores psico-socioeducativos, como valores, creencias y preferencias que, entre otros, pueden ser accionados por los docentes. Este concepto está definido en lo que él llama la Teoría del Erizo, constitutivo de tres círculos, que responden a: ser el mejor, tener pasión y generar motores. Lo pertinente de estos conceptos, estriba en un docente, por ejemplo, que debe tener un alto compromiso donde se manifieste un verdadero gusto por la docencia, asumida con pasión a fin de impactar la enseñanza.

Para Fullan (2011), las innovaciones educacionales se conducen con energizantes equivocados. Se aplica una racionalidad que al parecer impone una rendición de cuentas, cualidades individuales, tecnología forzada y una fragmentación. Pero, el rumbo sería crear: capacidad de construcción, trabajo en equipo e integración de estrategias. Argumentamos ahora, que así se estaría educando bajo la premisa de que esos conductores son efectivos para un cultura de crecimiento social que se fundamenta en el sentido común ya que lo contrario sería poner los caballos detrás de la carreta (Fuguet, 2011).

Las sentencias de atrapado en el barro con las ruedas girando, una talla no se ajusta a todos o bonito no es suficiente, sintetizan muchas consideraciones relacionadas con la violencia. Estamos como en una orfandad conceptual: científica, política, social y educacional. Orfandad que se evidencia, ahora, cuando a raíz de la Pandemia del Coronavirus las explicaciones sobre su origen, características, diagnóstico y tratamiento, fueron desorientadoras. En el caso de su impacto en los Sistemas Educacionales y de sus instituciones, fue evidente que la panacea no descansa en la conectividad exclusivamente electrónica. Sentimos la falta de algo, y ese algo se encuentra en la propia naturaleza del hecho pedagógico, que es la de un encuentro nutritivo entre el conocimiento, los medios, docentes y alumnos. 
Las sentencias anteriores, ilustrativas de la orfandad referida, son de críticas sobre las formas de investigar y conducir la educación por autores de renombre internacional. La primera es del Dr. David Turner, Glamorgan U., U.K.; la segunda, de Frances Schoonmaker de Teachers College de Columbia U., USA y, la tercera, de Larry Nucci, California U., Berkeley, USA. Si bien se refieren a las distintas formas de asumir y, en consecuencia, criticar el qué y el cómo se conduce la investigación educacional, lo hacen también con respecto a la vivencia escolar.

Sus ideas centrales estriban en cuáles motivaciones, principios y estrategias se fundamentan la investigación y la acción educativa. Es argumento aquí que sus planteamientos se adaptan a la crítica de la investigación y la acción de la Política, como objeto de estudio sistemático, científico y social. Especialmente, si la decisión política afecta la educación de un país, a sus instituciones y actores escolares.

Turner (2004), advierte que en materia de teoría de la educación, seguimos con las ruedas girando en el barro, lo que significa un ni para adelante ni para atrás. Al analizar factores multivariantes educacionales, reconoce que a pesar de contar con una cantidad de legislaciones que impactan al ambiente educacional, no lo hacen en la dirección esperada ya que entran en contradicción. Leyes sobre educación, trabajo, seguridad, salud, finanzas y transporte, generan un espacio político contradictorio, afectándose la garantía de que alguna institución escolar pueda satisfacer tantos contrastes al mismo tiempo.

Esta reflexión ubica el centro de atención en la dinámica de las políticas, ya que en educación no se trata sólo sobre metas de lo alcanzable sino sobre lo sustentable. Esto nos conduce a interpretar que el logro de ambas metas es razonablemente atribuido al manejo de bases teóricas adecuadas y a la coherencia en la coordinación como elementos claves para un servicio público de calidad. Imprescindible contar con instituciones sólidas, que posean un nivel de autonomía, aunque parcial. Autonomía para el individuo, la escuela y la sociedad en general.

Por su parte, Schoonmaker (2007), establece que desde inicios del siglo XX se cree que el uso de los hallazgos científicos pueda justificar y diseñar mejor la educación, con base en principios de la Psicología y con orientación a los métodos de la enseñanza. De un enfoque cuantitativo, amparado en procesos de 
experimentación, se ha llegado al uso de nuevas perspectivas metodológicas con visiones sociológicas y filosóficas. Así, se logra la integración entre investigación y la práctica pedagógica.

El arte y la ciencia de la investigación desde múltiples perspectivas paradigmáticas y la necesidad de entenderla como una práctica de una comunidad de maestros-investigadores en acción, son la fuerza para encontrar mejores bases teóricas, que orienten la práctica. Entonces, interpretamos que el maestro representa el trampolín para la conexión entre la investigación, la teoría y la práctica educacional. Es una poderosa arma de mejoramiento continuo por la diversidad de métodos que generen supuestos teóricos devenidos de la práctica. Argumentamos aquí, que impacta, también, aspectos de gestión, como son los procesos administrativos y los referentes gerenciales.

Para Nucci (2009), el problema es que lo bonito no es suficiente. Desde una Psicología Social centrado en el desarrollo moral del individuo, la escuela tendría mayores finalidades que formar un ser bueno, respetuoso, político, consciente e, incluso, conocedor, que sea visto como placentero y amado por los demás. Estas aspiraciones no abordan elementos sustantivos de reflexión profunda sobre el significado de la educación y, en forma especial, de la educación moral como misión escolar. Diremos aquí, que tampoco es suficiente una educación que se enfoca exclusivamente en la competencia laboral.

Por supuesto, la verdadera naturaleza u origen del desarrollo de los sujetos hoy, en tiempos de contrastes, crisis y caos, debe ir más allá de los convencionalismos para adentrarse en procesos de reflexión ética para toda la vida. El atender las necesidades de los individuos debe conducir al desarrollo de una plataforma de autoconfianza y disciplina para fortalecer la autonomía, la pertenencia, la competencia y la rectitud, como ingredientes del perfil del ser humano. Especificamos aquí, del ciudadano, del profesional y del servidor público, especialmente, de los agentes educacionales.

Así que movimientos van y vienen, innovaciones como epidemia. Y seguimos con grandes problemas educacionales, a pesar de contar con trabajos investigativos escolares, disponibles en repositorios en la Net. Muchos de esos problemas están relacionados con el ambiente en que se desenvuelve el sujeto, en donde se expresa la violencia con sus distintas caracterizaciones. 
Fase 2: selección de descriptores en un recorrido por las fuentes

La Sociedad de Educación Comparada e Internacional de Norteamérica (CIES), entidad asociada al Consejo Mundial de Educación Comparada, organiza su conferencia anual en donde participan aproximadamente unos 2500 investigadores, que pertenecen a diferentes países y son miembros de las diferentes SEC de otros países. Su operatividad académica radica en sus grupos de trabajo, denominados SIG's, es decir: Grupo de Interés Especial, que se consideran como líneas de investigación. La conferencia presenta una organización cuya responsabilidad corresponde a la sede universitaria seleccionada, pero apoyada por otras instituciones. Al mismo tiempo, recurre a un energizante orientador que en el CIES2019 fue sobre la sustentabilidad y en CIES2020, fue sobre educación más allá de lo humano hacia la Sympoiesis.

CIES2020 por razones del Convi19, se realizó como conferencia virtual. Su planificación varió considerablemente, por lo que se realizó la selección de descriptores con el programa de CIES2019 para este artículo. Para entonces se refieren 35 grupos de interés, algunos relacionados con el tema de este estudio, como los SIG's: Educación para la paz y prevención de violencia, Educación para la paz, Derechos humanos, Justicia social, Educación, conflicto y emergencias, Orientación sexual e identidad de género, Género y educación, Migraciones, Educación de refugiados, Raza y escolaridad, Educación no formal y popular, entre otros. Luego, se procedió a analizar CIES2020 a fin de ubicar información sobre el tema para destacar algunos datos complementarios.

Ahora bien, el recorrido por las sesiones SIG's2019, se procedió a la búsqueda de descriptores secuencialmente: violencia, paz, países, etc., a fin de ubicar las ponencias según los SIG'S, registradas en el programa de la conferencia. Estos descriptores pueden ser objeto de inventario ya que permiten ver términos, espacios, muestras, entre otros elementos investigativos. El resultado de este proceso permite aproximarse a una visión sobre cuáles bases, realidades y propiedades se estudia el problema de la violencia en sus peculiaridades. Cabe destacar que educación para la paz y prevención de violencia estaban 
reflejados en 28 grupos de ponencias y educación para la paz con 7 grupos. Hay que aclarar que esos grupos pueden estar divididos en subgrupos. Normalmente reúnen 3 ponencias.

CIES2020 por su condición de virtualidad se procedió a diseñar un espacio para presentaciones de libros, talleres, conferencias, etc., además de la sesiones SIG's. En este sentido se seleccionó en la sesión de libros, el presentado por: Papa, R. (2019). School violence in international contexts. Rosemary Papa (Ed.), Ed. Springer. Este recurso centra la atención en algunos casos de USA (Texas), Méjico, Palestina, Grecia, Nigeria, con búsqueda a reflexionar sobre nuevas exigencias del ente escolar en el marco de la Sympoiesis. Se hace referencia a problemas como: intimidación (bulling), disparos (shooting), forasteros (outsiders). Manifestaciones que generan miedo, rabia y sentimientos de estar bajo control. Hechos relacionados con la emulación de conductas, protocolos de prevención, gerencia escolar y salud mental. La solución se visualiza desde una educación para la paz y una escuela con visión de desarrollo social.

Desde la perspectiva de la Conferencia CIES2020, al asumir el constructo de Sympoiesis se permite, entonces, contar con un diferencial o reconcepto que permite la reorientación de palabras, términos y expresiones. Algunos autores las denominan valencias (Trifonas, 2000). Constituye una herramienta hermenéutica para ver la otra cara de la moneda que lleva a visualizar lo adverso como procedimental. Así que el concepto de autopoiesis, es entendido como cosas hechas o por sí mismos, autocreación ó autoorganización. Luego, Sympoiesis sería concebida como creación u organización colectiva. Se recomienda analizar la obra de Maturana y Varela (1973), sobre autopoiesis y cognición.

Desde estas ideas procedimentales, se pueden derivar criterios e informaciones de otras fuentes que pueden ser analizadas desde los planteamientos que permiten seleccionar conductores y descriptores para construir el análisis y reflexión categórica sobre el tema. 


\section{Categorías de análisis}

Fase 3: derivación de categorías de análisis

En internet se podrá conseguir variadas obras del tema. En Wikipedia, por ejemplo, se ilustra un buen material sobre ello. En resumen se visualizan aspectos en relación con violencia social, caracterizada desde varios ángulos, que incluye las siguientes expresiones:

- Se manifiesta en conductas con fines de dominación. El agente puede ser una persona o grupo. El paciente: el más desprotegido y, las razones se asocian a elementos: políticos, sociales, religiosos y culturales, en general. El factor radica más en el agente o ente agresor.

- Se observa en insultos, ofensas, amenazas y maltrato que generan daño físico, emocional, intelectual, mental y espiritual.

- El carácter valente puede observarse cuando los hechos vienen de un agente singular o institucionalizado. Se asocia a observar la causa en la condición animal de la especie humana, su condición gregaria y a la globalización, por redes sociales.

- Se puede clasificar de varias formas: visible: directa e, invisible: estructural y cultural $\mathrm{y}$, puede ser personal, interpersonal y colectiva:

- Personal: abuso del cuerpo, suicidio y mutilación.

- Interpersonal familiar: abuso sexual y explotación, secuestro, denigración de los miembros de la familia, en especial la pareja.

- Colectiva: institucionalizada en agendas y se manifiesta en guerras, crimen, terrorismo, miedo. Se asocia al Sistema: político, económico, organizacional, comunidad y del ente educativo. Se manifiesta también en una violencia ontoepistemológica con manejo del conocimiento, muy expresada en medios académicos.

El análisis con sentido de develar las valencias permiten un reto para observar el ambiente natural en el que se dan apreciaciones 
positivas y negativas, de acercamiento y lejanía, claro que lo negativo es barrera. Se puede destacar que:

- El estado de la persona presenta valores y necesidades, estados de equilibrio y tensiones.

- Existe un ambiente de violencia relacionada con la cultura cotidiana, en donde la persona no identifica como violencia algunos elementos contentivos en los hechos, escritos y discursos, es como invisible. Por ejemplo, entre libertad Vs. libertinaje, seguridad y dignidad humana.

- En las filosofías y sociopolíticas se puede desviar discursos como es el caso de justificar la paz lograda con violencia, cuyo efecto es de provisionalidad, precedido por degradación.

- También hay discursos como el de las grandes metas, que pretende dar a conocer beneficios que no se concretan o su valencia es lo contrario. Veamos: hombre de probeta, robótico, bomba atómica, que constituirán desechos.

Definitivamente hay un relación con el desarrollo psicosocial del sujeto. Veamos:

- Cultura, violencia y edad requieren de un desarrollo social y moral que centre la atención en las diferencias culturales para poder entender la transgresión a lo convencional.

- Hay una relación con el desarrollo psicosocial del sujeto. Las valencias adultos Vs. niños y jóvenes, lo convencional Vs. la instancia ética de la rectitud o el visualizar espacios placenteros, quedan al descubierto.

- Las estigmatizaciones, emulación y préstamos de discursos y la violencia de un Estado interventor, constituyen ingredientes de reflexión de la violencia invisible, pero institucionalizada colectivamente.

\section{Fase 4: Derivación desde CIES}

Desde la panorámica de los descriptores de CIES2019, se aprecia el deseo de establecer procesos de disminución de violencia. En este aspecto se seleccionan elementos comunes, 
como bases, realidades y propiedades en Sudamérica: Colombia, Ecuador, Perú, Chile, Argentina Uruguay y Brasil:

- Descriptores básicos: paz, sustentabilidad, activismo, acción colectiva, construcción, visión pedagógica, desarrollo humano, interculturalidad, inclusión, diversidad y ciudadanía. Constituyen bases para el discurso.

- Descriptores contextuales: idiosincrasia, género, trabajo infantil, refugiados, frontera, ruralidad, indígenas y bilingüismo. Constituyen realidades.

- Descriptores sustantivos: igualdad, oportunidad, desfavorecidos, calidad, acceso, elitismo, educación para el trabajo y segregación. Constituyen propiedades.

Siguiendo con CIES 2020, en su reflexión ante la pandemia manifiesta que su carácter catastrófico impone una reflexión-acción en educación, ambiente y cambios planetarios, cuyo centro de atención sea la reconceptualización del desarrollo a fin de hacerlo más sustentable, sin individualismo, nacionalismo, populismo $\mathrm{y}$, especialmente, sin limitantes teóricos y metodológicos, con vista en tecnologías alternativas. Es vernos en un escenario de pensamiento futuro post-humano que focaliza los derechos de mundos múltiples con ontoepistemología incluyente, tal como aceptación de la inteligencia artificial, pero en un mundo espiritual. Implica cambio de esquema para afrontar prácticas que permitan imaginar la vida y la educación en una tierra dañada.

Lo anterior nos lleva a seguir preguntándose si los proyectos como el de las de Guerrillas Escolares, idearios como lo cívicomilitar a conveniencia, organización de círculos como grupo de choques, entre otras expresiones de violencia institucionalizada, constituyen desviaciones que generan violencia. Al mismo tiempo que, preguntarnos qué pasará con los pacientes, ¿̇e sentarán en un pupitre de nuevo, cómo construir una nueva mentalidad escolar con prospectiva futura?

\section{La instancia de reflexión}

Del repertorio anterior se destacan elementos para una discusión sobre la violencia social, que permita la construcción de una interpretación desde fuentes que validen el repertorio, al mismo 
tiempo que contribuyan a formular una argumentación sustantiva sobre la temática. Algunos aspectos se vuelven neurálgicos en esta argumentación: el carácter valente y la reconsideración de conceptos, el concepto de violencia invisible, institucionalizada y colectiva $\mathrm{y}$, por supuesto, la violencia académica ontoepistemometodológica.

\section{El carácter valente y la reconsideración de conceptos}

El carácter valente de interpretación de la realidad de la violencia nos ubica en un proceso de reconsideración de conceptos. Al efecto Robertson (2010), de la Universidad de Bristol, Reino Unido, retoma el tema de las delimitaciones, reconfiguraciones y reordenamientos en los espacios políticos de Estado, ciudadanía y economía global. Incorpora conceptos como el de destatización, desnacionalización, descentralización y despolitización.

También, Rouco (2009) plantea que las palabras de siempre sirven para comprender problemas nuevos, como: conocimiento, Estado, sociedad, política, religión, y realidad, que en cotidianidad $\mathrm{y}$ en novedad han permitido formas humanas de convivir. Una reconsideración permitirá formular interrogantes sobre la facultad de limitar, condicionar, restringir y negar los derechos humanos, a la vida, libertad religiosa, pensamiento, conciencia, expresión y la enseñanza, sin que se quiebre su legítima ética. Establece que nadie puede disponer sin límite moral y jurídico de la familia, la libertad y de la asociación de los ciudadanos y nadie es dueño y señor de la sociedad, y, mucho menos, del ser humano.

Otro ejemplo de reconsideración lo constituye la visión del desarrollo, asociado a los que CIES2019 presenta como la necesidad de la sustentación. Una de las preocupaciones del Continente Africano, que impacta a la educación, es su futuro en manos de un desarrollo sustentable propio. Surgen interrogantes como: ¿Educación y desarrollo para quién? Académicos proponen una nueva teoría del desarrollo y crecimiento bajo una visión indigenista desde la condición valente de una crítica a modelos impuestos y a una valoración de su legado cultural.

La base científica del desarrollo no se desconoce, pero no es suficiente condición para la realidad africana. Esta opción se enraíza en su propia cultura y conocimientos de milenios y significa ir desde una concepción dominante y convencional hacia una que promueva el crecimiento local para dar soluciones 
amparadas en la creatividad, imaginación, recursos propios y la capacidad de gestión. Implica reorientar la educación para fortalecer competencias que logren procesos de unión entre tradiciones, conocimiento y experiencia local al proceso de desarrollo y, por supuesto, en unión con procesos de pensamiento y acción. Propósitos que se concretan en democracia.

La realidad de desarrollo africano, en muchos aspectos parecida a América Latina y el Caribe, estaría afectada por muchos condicionantes, especialmente el educacional. Desde este ángulo se demandarían políticas y programas que fortalezcan oportunidades, basadas en la diversidad lingüística y cultural y en las comunidades organizadas. Pero, América Latina podría, también, aprender de otros países.

Este planteamiento fue hecho por Fuguet (2015), al presentar el libro de Grimmett (2014), sobre la práctica del desarrollo profesional del maestro, realizado desde una perspectiva cultural e histórica en Australia. Esa práctica fundamentada en principios de mejoramiento educativo en escenarios de países en vías de desarrollo, como los suramericanos, podría reunir a investigadores, supervisores, directivos, administradores y asesores, para asumir claros principios para el perfeccionamiento educacional, al igual que a gobernantes, para establecer políticas acertadas según las necesidades de las localidades y centros educacionales.

\section{Violencia visible e invisible}

Desde el punto de vista de la reorientación conceptual con visión de perfectibilidad, exige una visión o camino, con efectos en la calidad educacional. Caminos que puedan evitar seguir atollados en el fango, vestir tallas ajenas, disfrazar de bonito lo incorrecto, eso sería como poner los caballos detrás de la carreta. Sin embargo, se hace necesario visualizar que algunos descriptores son amplios, cuyo carácter valente se evidencia. En el caso de la violencia visible (maltrato) vs. la invisible (cultural, institucionalizada), se da una relación peculiar. En definitiva esa división es didáctica que ayuda a comprender el problema en sus distintas facetas. Desde una filosofía del límite y lo fronterizo se puede interpretar tal división. El límite nos ubica en diferentes lados (Monreal, 2015; Trias, 1999; Morín, 2000).

El límite favorece un léxico de las ciencias como una herramienta de análisis, permite explorar dos líneas de interpretación marcado, 
caracterizado y definido como un proceso de inclusión y de exclusión. Estamos frente a una paradoja debido a que se construye como una estructura rígida, impermeable e intransitable, que genera exclusión. Sin embargo, la otra cara es que es vista como área de proximidad, de contacto e intercambio. Así, se da paso a una transformación que lleva a confluir el uno con el otro que se contraponen.

Desde el planteamiento anterior se puede observar una membrana muy delgada entre las violencia visible e invisible. Significa que hay necesidad de una revisión de las perspectivas para evitar posiciones excluyentes, sin perder al mismo tiempo los potenciales teóricos y políticos que estos conceptos revelan. Desde luego los pacientes de la violencia, pueden autogestionar salidas como en un proceso de autopoiesis, o sea de autogestión. Pero, sería una parcial solución ya que es necesario un proceso de construcción colectiva desde la interpretación de la Sympoiesis.

Abordar el conocimiento para ilustrar un diálogo que conduzca a visualizar al humano como ser del límite, lleva a recrear ese encuentro para ver al mundo con nuevos cauces y apoyarse en un espíritu con razón simbólica y metafórica para entender hechos que caracterizan al ser humano. Este encuentro se asocia a la idea de lo inesperado, que sorprende nuestra seguridad. Pero cuando brota debemos ser capaces de revisar las teorías y acciones. Revisión que se justifica ya que constituye una brecha entre la certeza y la incertidumbre. Definitivamente en la medida en que la violencia invisible genera efectos, se pone en evidencia y pasa a ser visible.

El concepto de límite y frontera no conduce a preguntarse: ¿Qué pasa en nuestras fronteras en América del Sur? Sin dejar de ver lo que pasa en el mundo, las realidades limítrofes de sus países presentan situaciones complejas y peculiares, que según los académicos, no se pueden analizar sin consideración teórica, metodológica y conceptual del desarrollo. Se aprecia que se está a las orillas de la aldea global frente doctrinas disímiles en una era de postconflictos, entre avances y contratiempos en materia de integración fronteriza, interdependientes al construir espacios de acuerdos, afectadas sus identidades y vida cotidiana, transfiguración de los Estados como efectos del crimen organizado, todo conectado o desconectado a asuntos socioeconómicos, políticos, culturales y educativos, exagerada migración infantil y 
juvenil, escasa ayuda humanitaria, abuso en comercio y consumo, doble nacionalidad, cierre de fronteras, posibilidad de conflicto armado, migración de la inversión extranjera, cambio climático y educación fronteriza (UFPS, 2018).

Esa realidad concreta de Colombia, Ecuador, Perú, Brasil y Chile, también, toca a Méjico y Costa Rica. Lógicamente, no se puede olvidar realidades en el pasado reciente de Salvador, Honduras y Nicaragua, el caso Cuba y Venezuela. El pasado que convierte a esos países en frágiles escenarios de vaivenes políticos y de violencia agenciada. En el caso de Venezuela, extremadamente descrita en una diáspora que supera las africanas, las de la fracción del Este Europeo y en otrora, la persecución contra el pueblo armenio y judío (Fuguet, 2020).

\section{La violencia académica}

Las citas anteriores amalgaman, como en cadena lo valente, la reconsideración de conceptos $\mathrm{y}$, por supuesto, la violencia invisible/visible, especialmente la concerniente en manejo del conocimiento en escenarios académicos. La situación se puede remitir a lo planteado por Tomas Armstrong (2006), en su obra Las Mejores Escuelas, en donde se abre un camino para demostrar que la investigación, sobre un apropiado desarrollo humano, puede documentar mejor la práctica educacional. Parte de una diferencia entre lo se denomina el discurso del logro académico vs. el discurso del desarrollo humano. Una aclaración necesaria, lo académico no tiene nada de malo. Pero, a veces se percibe muy estructurado y con una visión estrechamente de medición de resultados de los aprendizajes descritos en los planes curriculares y validados por instrumentos evaluativos. Detrás de ello hay una concepción de currículum como plan y no de currículum como experiencia. Ambos enfoques tienen diferentes características en aproximadamente unos 12 supuestos que rigen el ambiente escolar. Para este ensayo, se seleccionan algunos, por lo extenso del discurso. Según él, el logro académico funciona:

- Como en un ambiente de arriba hacia abajo en la que las personas con gran poder imponen programas, procedimientos y políticas a aquellos de menos poder. Luego, se espera que los maestros, directores, administradores y supervisores asuman un similar discurso al que ostentan el poder: presidentes, 
gobernadores, legisladores y empresarios. Las aristas de interpretación es que en los grandes países desarrollados la influencia de este discurso se atribuye más a políticas públicas que orientan su acción a satisfacer el futuro desarrollo económico y asegurar trabajo al educando.

- La realidad educativa generada por esa práctica tiene consecuencias ya que se: a) eliminan áreas de estudios que darían una amplia educación para experimentar éxito y una vida llena de satisfacciones; b) obvian experiencias que no sean validadas por test; c) generan la copia, el plagio, uso de sustancias ilegales y promueven la manipulación de la data; e) transfieren el control del currículum a otras instancias; f) aumentan el estrés, la repetición y la deserción; g) desconocen las diferencias individuales de cultura y estilos de aprendizaje; h) desconocen los valores y expectativas del aprendizaje y, i) permiten inapropiadas prácticas instruccionales.

- Ello afecta a la escuela y, así, toma significado el discurso alterno, el humanista que se caracteriza por: a) atender a la felicidad del ser humano en su totalidad, como principio cardinal y acciones con significado; b) favorecer un currículum flexible que ofrezca opciones enriquecedoras y, c) permitir un esfuerzo coordinado de la comunidad educativa inspirado en un pensamiento creativo. Una síntesis, sería que el discurso del desarrollo humano envuelve un ambiente de aprendizaje creativo que ofrece al estudiante opciones significativas de lo que se aprenderá en la vivencia de la experiencia escolar para consolidar el desarrollo de una propia y única versión del ser humano en su totalidad.

Definitivamente ese cuadro de Armstrong es demostración del estar atollados en el fango, el de contar con una sola prenda para vestir y el centrarnos en asuntos bonitos pero no sustantivos de la educación familiar y escolar. Otra fuente de validación se encuentra en los planteamientos que realizan William Schubert y colaboradores (2002), en su obra Curriculum Books, cuando después de haber analizado múltiples visiones curriculares, incluso venidas de rutas metodológicas propias del activismo 
político o social, aceptan que históricamente grandes personalidades han servido para perpetuar intereses mitológicos e ideológicos de grupos de dominación social, afectándose las vidas de educadores, estudiantes e instituciones.

Por supuesto, el planteamiento anterior tiene aristas científicas al desconocer logros en el conocimiento de una innovación educativa, al pretenderse usar criterios parciales de investigación educacional tal como lo describe Schoonmaker. También, abarca aspectos de crítica a las políticas y a las legislaciones en búsqueda constante de la perfectibilidad personal y social, en democracia. Nos encontramos ante una tensión creativa que nos impone cambios drásticos en la función de la familia y la escuela. Lógico, que la educación debe seguir con su responsabilidad original, pero con nuevas prácticas pedagógicas cónsonas con los tiempos. Situación que exige un cambio adecuado en las políticas públicas educacionales.

\section{La violencia política}

Ahora, se impone el atender una población afectada en sus valores humanos, ciudadanos y republicanos como legado cultural de la violencia, en sus distintas expresiones, dimensiones y particularidades. Así, que la familia y la Escuela, como instituciones no pueden dejar de hacer una labor de reeducación. Lo que implica procesos de revitalización. Ahora: ¿Cómo lograr una persona correcta, ciudadano demócrata si no se vivencia escenarios nutritivos en lo social y familiar de democracia al interno? Mejor: ¿Cómo pueden la familia y la escuela relacionarse para ese logro? Se tendrá éxito si hay participación, condición necesaria para el compromiso. Muchas de las innovaciones, vienen como epidemia, sin fundamento. Así, que hay que tener sentido común al diseñarlas, para no poner los caballos detrás de la carreta.

Los argumentos anteriores nos lleva a pensar en la relación de dos espacios: el político y el educacional. El 
Estado interventor y no promotor, afecta la calidad en todas sus expresiones. Esa intervención disminuye la posibilidad de formar cerebros calificados y evita que las instituciones educativas sean entes inteligentes, es decir que aprendan. Se violan sus bases filosóficas y pedagógicas y limita su acción a un simple servicio público. Lo peor de ese servicio, es que nutre ideologías, que distorsionan el verdadero rol del Estado y de la sociedad en general. Para sus fines, se manejan erradas concepciones sobre currículum, evaluación y supervisión educacional. Necesario sus reconsideraciones, ya que de lo contrario seguirán adoptándose políticas públicas y legislaciones que afectan la calidad y, con ello, la supuesta igualdad y equidad.

Ante ello recordamos lo planteado por Turner en cuanto a que tantas exigencias políticas y legislativas no pueden simultáneamente se respondidas y respetadas por las organizaciones escolares, de salud y de transporte, Etc., entes de la vida cotidiana. Reflexión sobre la dinámica de las políticas que desconocen que en educación se trata de metas de lo sustentable y no sólo de lo alcanzable. Ello se relaciona con el manejo de bases teóricas adecuadas y con la coherencia en la coordinación de elementos claves para un servicio público de calidad. Imprescindible, también, contar con autonomía, aunque parcial, para el individuo, la escuela y la sociedad en general.

Ante los argumentos anteriores, el ideario de la democracia lenta de Clark and Teachout (2012), permite centrar la atención en algunas consideraciones, entre ellas:

- La democracia rápida, envuelta en frases eufemistas, demagógicas y populistas entre otras manifestaciones, encaran una democracia que se fundamenta más en sus adjetivos y no en lo sustantivo. Democracia bonita o fuerte no es suficiente, emulando a Nucci, en su expresión ya referida. 
- La democracia lenta no es un llamado para reuniones largas o tomar más tiempo en las decisiones. Es un recordatorio de la atención necesaria, con la toma de decisiones comunitarias. $\mathrm{Al}$ igual que la comida rápida, la democracia rápida ofrece un producto fácil de usar, pero deja a los ciudadanos desnutridos e insatisfechos.

- Luego, se puede interpretar que hay necesidad de reaprendizaje sobre lo que significa la democracia. Se trata de luchar y ganar. El sólo hablar empeora, los problemas se tornan complejos, se reduce el entendimiento de los ciudadanos, la participación toma tiempo y el gobierno es lento en soluciones. Desde luego lo bonito no es suficiente.

- Las soluciones a nivel nacional son casi inviables, hacerlo a nivel local es más factible. Permite dar soluciones del mundo real para el mundo real de problemas. La clave es el saber decidir cómo decidir, con uso de un modelo de inventar nuevas soluciones juntos. Se sintetiza así, la lucha hacia una educación de calidad del ciudadano. De lo contrario, tendremos desnutridos e insatisfechos educacionalmente hablando, al no proporcionarle los nutrientes que dote del control del conocimiento y la experiencia. Nutrido, se podrá adecuadamente participar en el desarrollo de un país, con criterio de independencia y autonomía de pensamiento, instancia ética y conducta moral. Este es el rol escolar. Logro que debe iniciarse tempranamente en la escuela.

Esa instancia nutritiva va unida a una educación familiar y escolar de calidad. Definitivamente sería como espacios para evitar la violencia social en todas sus expresiones. Necesario inspirarse en la Pedagogía, en sus disciplinas propias y las ciencias auxiliares que, en conjunto, han producido las estructuras conceptuales orientadoras de la acción educativa. La violación a esas estructuras, es negación de los principios filosóficos, científicos y sociales que los soportan. En este 
caso, destacamos que currículum es la experiencia vital que el sujeto vive bajo el auspicio escolar. Esta definición desplaza otras que restringen su concepción como disciplina, sistema y especialmente a currículum como plan o programa.

Pero, la calidad es compleja, requiere de visión integral. Según Fuguet (2018), llama a ofrecer una experiencia que desarrolle autonomía de pensamiento, criticidad y una acción coherente en el estudiantado. Conlleva a: a) integrar filosofía, psicopedagogía, socio-antropología y administración, al desarrollo curricular, de la didáctica, la evaluación y la supervisión escolar; b) concretar políticas reales y no demagógicas, y, c) manejar una perspectiva del docente, desde su formación en las instituciones universitarias y su desarrollo profesional durante todo su ejercicio. Esta calidad se basa en procesos de docencia, dirección y supervisión.

Desde esa perspectiva se pueden plantear ideas sobre una adecuada participación ciudadana en general y en educación, particularmente. Participación integraría esfuerzos familiares y escolares para la prevención de la violencia. Pero la participación no se logra con una varita mágica. Es compleja, puesto que requiere de visualización, conciencia, experticia y fundamentalmente, instancia ética. La participación no se trata de lograr la aprobación de programas y justificar decisiones de arriba hacia abajo, tampoco de intervencionismos al crear instancias con unos ciudadanos para el control de otros. Así sería un proceso improductivo e insatisfactorio que contribuye con la desnutrición ciudadana. Se trata de tomar decisiones con el manejo de un espectro de opciones, que puedan orientar al Estado, a las instituciones y a gestores para tomar mejores decisiones.

Por lo general hay discursos que contradicen la realidad vivida en las instituciones. Pero, los verdaderos maestros, los gestores y los supervisores, al fin y al cabo, gobiernan las escuelas. Según Conley (2003), a pesar de que la intención, como agenda política, es la intervención en asuntos educacionales, no ha sido del todo aceptada y aplicada. Plantea que ese reto de gobierno en las escuelas es de los 
docentes, quienes deben estar preparados para asumirlo. Podemos, entonces, argumentar que el docente es el cocinero de una receta (currículum como plan), pero es el modelaje de él y demás miembros de la comunidad quienes guían en definitiva la experiencia escolar. Este concepto es extendido a la familia, ya que sus miembros necesitan un encuentro para evitar y manejar experiencias de violencia social.

Retomando el aspecto de los motores, energizantes y conectores de Collins y de Fullan, como constantes flujos de ganancia social, destacamos que los valores, creencias y preferencias pueden ser accionados para un mejor aprendizaje. Pero, especialmente para logar habilidades de compromiso de todos. Compromiso para realizar la labor siendo los mejores, con pasión y determinación. El compromiso manifestado por los padres, docentes y otros miembros de la comunidad es estímulo eficaz para lograr que los estudiantes controlen la violencia manifiesta en sus etapas de desarrollo humano. Meta que se consolida al tener energizantes como capacidad de construcción, trabajo en equipo e integración de estrategias.

\section{Violencia ontoepistemometodológica}

Desde el ámbito de la violencia organizada retomo un elemento valente para el tema, relacionado con la realidad universitaria. Según Trifonas (2000), asume que existen principios que rigen una cultura universitaria. El cuestionamiento, desde un análisis crítico postmoderno, conlleva a desconstruir y reconstruir estructuras conceptuales. Pero, ello responde al principio de razón como base de la racionalidad moderna. La clave estaría en una apropiada visión de la actuación desde el interno de uno mismo o sea autoreflexivamente. Ese argumento lleva a la redefinición de la responsabilidad universitaria, ante el manejo de los procesos de informatización, ligado al lenguaje, a la estética y a la ética. Así que valores como profesionalismo y disciplinariedad, orientarían una comunidad de cuestionamiento con un discurso intrainstitucional.

Oportuno recordar el planteamiento sobre la investigación desde múltiples perspectivas paradigmáticas, que debe ser asumida como una práctica de una comunidad de investigadores en acción, para constituirse en fuerza con las bases teóricas que orienten la práctica. La investigación, unida a la práctica educacional, es un 
arma de mejoramiento continuo por la diversidad de métodos y supuestos teóricos. Una reconsideración de conceptos, también aplica para otros aspectos además de métodos y supuestos, entre ellos variedad de: fuentes, medios, formas de documentación, diagramación, esquemas, protocolos y normas. Ello permitirá libertad de expresión en la producción intelectual. Al mismo tiempo que desprenderse un poco de normas para satisfacer formas de acción y comunicación con criterios no acordes con una instancia interna de la reflexión.

El discurso academicista ha producido presión y estrés, al mismo tiempo que desafíos. A veces se imponen enfoques que nos mantienen atollados en el fango, sin poder ver a otros lados para encontrar otros nutrientes. La carrera de los docentes e investigadores radica más en protocolos de investigación asociados a evidencias empíricas, que son prendas de vestir de una sola talla y se constituyen un desmedro de otras fuentes y criterios de indagación. Luego, se imponen mecanismos rigurosos de publicación que traducen ese modelo cientificista. Al no dejar espacios para diferentes formas de creación del conocimiento y reflexión sobre la experiencia, se produce una situación de violencia bien definida en sus caracterizaciones ontológicas, epistemológicas y metodológicas. Ello representa un desconocimiento de la ética institucional.

No se niega elementos de unificación de criterios para la evaluación de la producción intelectual, pero no se puede pedir identificar toda la realidad y su indagación a criterios exclusivamente de las ciencias duras y fácticas. Hay mecanismos propios de la filosofía, la teología, las artes y las humanidades, así como en toda acción de acercamiento al conocimiento, que constituyen un abordaje creativo. Su comunicación responde a razón abierta. Razón que atiende al carácter cognoscitivo de la persona en sus distintas expresiones. Expresiones sociales, políticas, científicas, religiosas, de creencias, artísticas, entre otras que demandan flexibilidad de temáticas, fundamentos, métodos y recursos discursivos variados.

Razón abierta responde a un dialogo y es la idea central de Joseph Ratzinger: Benedito XVI (2005, 2012). Él parte de dos factores: la formación de una sociedad global interdependiente y la posibilidad humana de crear y destruir, que suscita una cuestión del control racional. Toma fuerza el que las culturas encentren 
fundamentos de convergencia para regulaciones racionales. Argumenta que: a) la interpenetración cultural ha quebrado las certezas éticas y que el conocimiento, especialmente el científico, no genera una ética, pero afecta certezas morales; b) la responsabilidad de la ciencia, la filosofía y otras disciplinas, se demuestra con posturas críticas sobre sus conclusiones acerca del origen y propósito de la existencia, que implica razón de mentalidad abierta a dimensiones amplias de la verdad.

Luego, se demanda del poder la garantía del uso razonable de la ley a fin de controlar la arbitrariedad. Plantea que el conocimiento científico, social, filosófico, etc., debe preguntarse por los orígenes del poder y las leyes. También, por los derechos humanos, que en esencia son valores intocables, pero violados por: guerras, opresión, terrorismo y fanatismo religioso. La ciencia, por ejemplo, aparenta traer amenazas. Ejemplos son los seres de probeta como producto, que a la larga serán desechos y la bomba atómica. Luego, sería mejor que la razón sea vigilada. Aún más, que la razón, la política y la religión se regulasen recíprocamente. Ante lo anterior, otros saberes son necesarios.

Autores que recientemente han disertado sobre la razón abierta, como Pérez (2019), quien plantea que, en el mundo contemporáneo, existe desconfianza de los recursos cognoscitivos del hombre. Situación que conduce a conformarse con verdades parciales, sin develar el sentido último de la vida humana. Por otra parte Barrio-Maestre (2013) determina que la noción de investigar necesita de variados ámbitos de mutua interrelación. Asimismo, Bellini (2018), manifiesta que la ética, la antropología, la política y la educación, han afrontado los mismos conceptos de razón en la formulación científica desde el valor de distintas ramas del saber. También, Fraguas (2021), dice que ante el despliegue de la pandemia, por ejemplo, se evidencia la necesidad de acudir a otras disciplinas complementarias como las humanísticas, que pueden permitir aproximarnos a respuestas apremiantes.

\section{CONCLUSIONES}

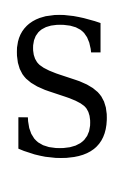
e ha abordado aspectos referidos a la violencia, desde variadas perspectivas. Factores sociales, educacionales, políticos y culturales, entre otros aspectos, entran en juego con aristas a veces contradictorias. En consecuencia, se ha 
destacado la complejidad de la temática debido al manejo de discursos valentes que conllevan a observar pros y contras en los idearios que encierran. La visión internacional dada al análisis ofrece un marco pluridisciplinario y multicultural para una descripción de la situación del problema mundialmente.

Se ha descrito parte del problema desde las investigaciones realizadas por investigadores internacionales, miembros de las Asociaciones de Educación Comparada de sus respectivos países y que participaron en los encuentros CIES2019 y CIES2020, con estudios en la temática desde diferentes grupos de interés prioritarios con impacto en educación: paz, prevención, derechos, justicia social, conflicto, emergencias, género, migraciones, refugiados, raza, escolaridad y educación no formal y popular.

La situación de nuestros países suramericanos no escapa a la situación de globalidad. Se identifican descriptores como: paz, sustentabilidad, activismo, acción colectiva, construcción, visión pedagógica, desarrollo humano, interculturalidad, inclusión, diversidad, ciudadanía, idiosincrasia, género, trabajo infantil, refugiados, frontera, ruralidad, indígenas, bilingüismo, igualdad, oportunidad, desfavorecidos, calidad, acceso, elitismo, educación para el trabajo y segregación.

Ante esa realidad contradictoria, el análisis de reconsideración de términos, conceptos y discursos demuestra la necesidad de lograr una aproximación interpretativa, comprehensiva y abarcadora de la problemática de la violencia compleja y posibles soluciones, como reto de la educación. Ello permitió observar discursos que nos mantiene atollados sin salir del fango, vestidos con prenda ajena y viéndonos en discurso bonitos, pero no sustantivos y nutritivos educacionalmente. Discurso que hacen manejar conectores errados de innovación y que permiten dejar los caballos detrás de la carreta. Su efecto es quedarnos en una orfandad conceptual.

Acerca de los conectores errados, se plantea que bien concebidos podrán favorecer un aprendizaje significativo si se convierten en motores o energizantes de compromiso familiar, social y escolar en pro de una educación preventiva y correctiva de la violencia. En este sentido la gestión de la familia y la Escuela, como instituciones sociales, es destacada en su rol de reeducación. Especialmente en el logro de una persona formada con rectitud en el pensar y obrar humanamente. Esta dimensión se 
relaciona definitivamente en términos de Sympoiesis, desde la perspectiva de una construcción colectiva que brinde satisfacción individual y social.

Dentro de la violencia invisible/visible, se destaca la violencia ontoepistemometodológica con planteamientos de razón abierta y flexible, para poder contar con un espectro de inclusión del pensamiento, de la acción y de la producción intelectual, en las variadas perspectivas del mundo académico. Constituye un espacio intrapersonal e intrainstitucional en que toma sentido la construcción colectiva del conocimiento y la correcta interpretación de la experiencia cotidiana o especial vivida.

\section{REFERENCIAS}

Armstrong, Th. (2006). The Best Schools. Alexandria, VI: ASCD, 13. The Best Schools | Thomas Armstrong, Ph.D. (institute4learning.com)

Asabere-Ameyaw, A. ET All. (2014). Indigenist African Development and Related Issues. Rotterdam: Sense Publishers. Review: [Untitled] on JSTOR

Barrio-Maestre, J. (2013). Circularidad fe razón en Joseph Ratzinger. En Pensamiento y Cultura, Vol. 16-1: 67-201

Belini, L. (2018). Razón y Fe: historia y perspectivas, una mirada reflexiva. En Iza Villacis V. A. (Ed.), Persona, Educación y Filosofía (11-43). Quito: Abya-Yala.

Benedicto XVI (2005). Dialogo entre la Razón y la fe. Buenos Aires: Diario La Nación. https://www.lanacion.com.ar/cultura/dialogoentre-la-razon-y-la-fe-nid 704223/

Benedicto XVI (2012). El Año de la fe: la razonabilidad de la fe en Dios. Roma: Audiencia General del 21 de Nov. 2012

Comparative e International Education Society (2019) Program 63 Annual Conference. San Francisco. CIES 2019 - San Francisco - April 14-18, 2019

Comparative e International Education Society (2020) Program 64 Annual Conference. Miami Welcome - CIES 2020

Clark, S. and W. Teachout (2012). Slow Democracy, Vermont: Chelsea Green Publishing. Slow Democracy: Rediscovering Community, Bringing Decision Making Back Home - Susan Clark, Woden Teachout - Google Libros

Conley, D. (2003). Who governs our schools: changing roles and responsibilities? New York, Teachers College Press. ERIC ED476169 - Who Governs Our Schools? Changing Roles and Responsibilities. Critical Issues in Education Leadership. 
Collins J. (2002). Empresas que sobresalen. Bogotá: Paidos

Fuguet, A. (2015) Book Review. In Comparative Education, Vol. 59, No. 2. Chicago: University of Chicago Press, 371-372. Review: [Untitled] on JSTOR

Fuguet, A. (2018). Revolución y Caricatura. Caracas: FEDUPEL. 025-Revolucion-y-Caricatura.pdf (upel.digital)

Fuguet, A. (2020). De la Educación de la Diáspora a la Educación de Postconflicto y Emergencias. En Revista de Investigación, № 99 (44): 13-45. https://www.bing.com/search?q=revista +investigación+Fuguet+diáspora+2020\&qs $=n \&$ form $=Q B R E \&$ $\mathrm{sp}=-1 \& \mathrm{pq}=$ revista +investigación +fuguet+diáspora $+2020 \& \mathrm{sc}$ $=0-42 \& s k=\& c v i d=3 B C B C E 4 C 124940$ C9BA8869C7D6208E78

Fullan, M. (2011). Choosing the wrong drivers for whole system reforms. Victoria: Centre for Strategic Education, Seminar Series, No. 204. 13396088160.pdf (michaelfullan.ca)

Fraguas (2021). Razón y Fe. En Revista Hispanoamericana de Cultura 287: No. 1449: 77-86. razón y fe - Bing

Grimmett, H. (2014).The Practice Teachers Professional Development: A cultural historical approach. Rotterdam: Sense Publishers. The practice of teachers' professional development: a cultural-historical approach - Monash University

Maturana, H., and F. Varela (1980). Autopoiesis and Cognition: the Realization of the Living. Robert S. Cohen and Marx W. Wartofsky (Eds.), Boston Studies in the Philosophy of Science 42. Dordrecht: D. Reidel Publishing Co

Monreal, C. (2015). Summer School 2015: Exploraciones entre fronteras, miradas entre dos orillas. U. Florencia, en el marco del proyecto GENDERCIT: Programa People 2007-2013)

Morín, E. (2000). Los Siete Saberes de la Educación del Futuro. Caracas: UNESCO-IESAL, FACES-UCE, CIPOST. Avant-propos (ideassonline.org)

Nucci, L. (2009). Nice is not enough. Upper Sandler River: Pearson

Papa, R. (2019). School violence in international contexts. Rosemary Papa (Ed.), California: Springer School Violence in International Contexts - Perspectives from Educational Leaders Without Borders | Rosemary Papa | Springer

Pérez, F. (2019). Razón y fe se complementan. SIC. Fe y Razón se complementan | Agencia SIC

Robertson, S. (2009). The new spatial politics of re - bordering and re - ordering the state - education - citizen - relation for the global economy. Istanbul: XIV World Congress of Comparative Education Societies 
Rouco, A. (2009). Iglesia, Sociedad y Política. Madrid: Colección Cartas Pastorales del Sr. Cardenal - Arzobispo de la Archidiócesis de Madrid. Discurso del Cardenal Rouco en la sesión de apertura de la reunión plenaria de la Conferencia Episcopal | Agencia SIC

Schoonmaker, F. (2007). Stuck in the Mud with the wheels spinning, one size doesn't fit al all: reopening discussion of the researchpractice connection. In Theory into Practice, 46 (4), LEA: 164-171

Schubert W. Et Al. Curriculum Books: the First Hundred Years. New York: Peter Lang Publishing Inc

Trias, E. (1999) La Razón Fronteriza. Barcelona: Destino

Trifonas, P. (2000). The Ethics of Writing: Derrida, Deconstruction and Pedagogy. Lanham, Ma. Bowman \& Littlefield Publishers INC

Turner, D. (2004). Theory of Education. London: Continuum Studies in Education

Universidad Francisco de Paula Santander (2018). Estudios Transfronterizos: impactos y retos en América Latina. O. Sierra Coordinador, Cúcuta: Editorial UFPS 